H. Kumura

Nagoya Math. J.

Vol. 138 (1995), 1-18

\title{
THE DIRICHLET PROBLEM AT INFINITY ON HADAMARD MANIFOLDS
}

\author{
HIRONORI KUMURA
}

\section{Dedicated to Professor Hideki Ozeki on his sixtieth birthday}

\section{Introduction}

Let $M$ be an $n$-dimensional Hadamard manifold, that is, a complete simply connected $C^{\infty}$ Riemannian manifold with nonpositive sectional curvatures. Making use of geodesic rays, Eberlein and O'Neill [11] constructed a compactification $\bar{M}=M \cup S(\infty)$ of $M$ which gives a homeomorphism of $(M, S(\infty))$ with the Euclidean pair $\left(B^{n}, S^{n-1}\right)$. In this paper we shall study the asymptotic Dirichlet problem for the Laplace-Beltrami operator, which is stated as follows:

Problem. Given a continuous function $\varphi$ on $S(\infty)$, find a harmonic function $u \in C^{\infty}(M) \cap C^{0}(\bar{M})$ such that $\left.u\right|_{S(\infty)}=\varphi$.

The maximum principle implies that $\varphi$ uniquely determines $u$ if it exists.

This problem has been studied by several authors (see [2], [3], [4], [7], [8], [9], [14], [15], [16], [20] and [21]). Choi [9] solved this problem for rotationally symmetric manifolds with the decay of sectional curvatures faster than - $A \rho^{-2}\{\log \rho\}^{-1}$ outside a compact subset, where $A>1$ is a constant and $\rho$ is the distance from a fixed point. In [3] and [21], Anderson and Sullivan independently settled this problem affirmatively for the manifold with the sectional curvatures bounded between two negative constants. In relation with this result, the following question is proposed by Yau [22, p. 14]: if curvatures of $M$ are bounded above by a negative constant, can one find nontrivial bounded harmonic functions defined over the manifold? From this point of view, as a first step, we shall attempt to relax the pinching condition for the curvatures which is imposed in Anderson [3] and Sullivan [21]. A work in this direction was actually done by Hsu and March:

Received April 1, 1994. 
THEOREM 1.1 ([15], [14]). Let $M$ be an Hadamard manifold of dimension $n \geq 3$ and assume either (a) there are constants $\alpha>2,0<\beta<1$ and $L>0$ with $\alpha(1-\beta)>2$ such that

$$
K_{M}(x) \leq-\alpha(\alpha-1) \rho(x)^{-2}
$$

and

$$
\operatorname{Ric}_{M}(x) \geq-L \rho(x)^{2 \beta}
$$

for all $x \in M$ outside some compact subset; or (b) there are constants $U>0$ and $L>0$ with $U^{2} / L>(n-1) / 2$ such that

$$
K_{M}(x) \leq-U^{2}
$$

and

$$
\operatorname{Ric}_{M}(x) \geq-L^{2} \rho(x)^{2}
$$

for all $x \in M$ outside some compact subset, where $\rho(x)=\operatorname{dist}(o, x)$ and $o \in M$ is a fixed point of $M$ and $K_{M}(x)$ (resp. $\operatorname{Ric}_{M}(x)$ ) denotes the sectional curvature of $M$ (resp. the Ricci curvature of $M$ ). Then one can solve the asymptotic Dirichlet problem for the Laplace-Beltrami operator.

In this paper, we shall generalize Theorem 1.1 and prove the following:

THEOREM 1.2. Let $M$ be an Hadamard manifold of dimension $n \geq 3$ and assume either (a) there exist constants $l>2, C>0$, and $\theta>0$ such that

$$
K_{M}(x) \leq-l(l-1) \rho(x)^{-2}
$$

and

$$
\operatorname{Ric}_{M}(x) \geq-C \rho(x)^{l-2}\{\log \rho(x)\}^{-\theta-1}
$$

for all $x \in M$ outside some compact subset; or $(b)$ there exist constants $a>0, C>0$ and $b \in(0, a)$ such that

$$
K_{M}(x) \leq-a^{2}
$$

and

$$
\operatorname{Ric}_{M}(x) \geq-C e^{b \rho(x)}
$$

for all $x \in M$ outside some compact subset. Then one can solve the asymptotic Dirichlet problem for the Laplace-Beltrami operator. 
To prove Theorem 1.1, Hsu and March used a probabilistic approach. Their proof requires some lower bound of the Ricci curvature which guarantees the conservative law for the heat equation to hold; see [15, Remark 3]. This is the reason they imposed the condition $\operatorname{Ric}_{M}(x) \geq-C\left(\rho(x)^{2}+1\right)$. Indeed, Li and Yau [18] showed that if the Ricci curvatures on a complete Riemannian manifold $M$ satisfy $\operatorname{Ric}_{M}(x) \geq-C\left(\rho(x)^{2}+1\right)$ for some positive constant $C$, then a nonnegative solution of the heat equation on $M$ is uniquely determined by its initial data and therefore the minimal heat kernel $p(t, x, y)$ satisfies $\int_{M} p(t, x, y) d V(y) \equiv 1$. On the other hand, Azencott [6] showed that this curvature bound is almost sharp. To be precise, he showed that if $M$ is an Hadamard manifold whose sectional curvatures are bounded from above by $-C \rho(x)^{2+\varepsilon}$ for some positive constants $C$ and $\varepsilon$, then the minimal heat kernel $p(t, x, y)$ satisfies $0<\int_{M} p(t, x, y) d V(y)<1$ for $t>0$ and hence a solution of the heat equation on $M$ is not uniquely determined by its initial data. Obviously, Theorem 1.2 includes the case that $M$ is not stochastically complete.

On the other hand, we shall prove Theorem 1.2 by a more geometric method than Hsu and March's one. Indeed, we shall construct $C^{2}$-superharmonic and subharmonic functions with given boundary value on $S(\infty)$, making use of the Schoen's technique in [4]. In our proof, we shall use only the comparison theorems in Riemannian geometry and the maximum principle.

This paper is divided into five sections. In Section 2, a key estimate for the proof of Theorem 1.2 will be obtained by using the comparison theorem in Riemannian geometry. Section 3 is devoted to proving Theorem 1.2. In Section 4, we shall state a generalization of Theorem 1.2 to the Dirichlet problem for harmonic maps. Some examples of manifolds will be given in Section 5 .

Acknowledgement. The author would like to express his gratitude to Professor Atsusi Kasue for his valuable suggestion and warm encouragement.

\section{A key estimate}

To begin with, we choose a fixed point $o \in M$ and identify $S(\infty)$ with the unit tangent sphere $S_{o}^{n-1}(1)=\left\{u \in T_{o} M ;\|u\|=1\right\}$ via the exponential map at $o$, where we assume that $S_{o}^{n-1}(1)$ has the standard sphere metric. Let $\varphi$ be a Lipschitz function on $S_{o}^{n-1}(1)=S(\infty)$ and extend it radially along the rays from $o$ to a function on $\bar{M}-\{0\}$, with boundary data $\varphi$ on $S(\infty)$. Let $\chi:[0, \infty) \rightarrow R$ be a 
fixed $C^{2}$-function such that

$$
\begin{gathered}
\chi(t)= \begin{cases}1, & 0 \leq t \leq 1, \\
0, & 2 \leq t,\end{cases} \\
\chi^{\prime}(t) \leq 0, \quad t>0,
\end{gathered}
$$

and

$$
\chi^{\prime \prime}(t) \geq 0, \quad 7 / 4 \leq t \leq 2 .
$$

As in [4], we define a function $A(\varphi)$ by

$$
A(\varphi)(x)=\frac{\int_{M} \chi(\rho(x, y)) \varphi(y) d V(y)}{\int_{M} \chi(\rho(x, y)) d V(y)},
$$

where $\rho(x, y)=\operatorname{dist}(x, y)$ and $d V$ denotes the Riemannian measure on $M$. We note that $A(\varphi)$ is a $C^{2}$-function on $M$ with boundary value $\varphi$ on $S(\infty)$. Then we have the following:

Lemma 2.1. The Laplacian of $A(\varphi)$ satisfies

$$
|\Delta A(\varphi)|(x) \leq C_{1}\left(k(x)^{2}+1\right) \cdot \sup _{y \in B_{x}^{(2)}}|\varphi(y)-\varphi(x)|,
$$

where

$$
-k(x)^{2}=\inf \left\{\frac{\operatorname{Ric}_{M}(u, u)}{(n-1)\|u\|^{2}} ; 0 \neq u \in T_{q} M, q \in B_{x}(2)\right\}
$$

and $C_{1}$ is a constant depending only on $\chi$ and $n=\operatorname{dim} M$.

Remark 1. In [4], Anderson and Schoen took an auxilialy function $\chi$ satisfying (1) and showed that

$$
\left|D^{2} A(\varphi)\right|(x) \leq C_{1}^{\prime} \cdot \sup _{y \in B_{x}(2)}|\varphi(y)-\varphi(x)|,
$$

when the sectional curvatures of $M$ are bounded between two negative constants. Here, $D^{2} A(\varphi)$ is the Hessian of $A(\varphi)$ and $C_{1}^{\prime}$ is a constant depending only on the choice of $\chi$, the curvature bounds and ambient geometry of $M$ (see [4, p. 437]).

Proof of Lemma 2.1. Direct computation shows that

$$
\Delta(A(\varphi))\left(x_{o}\right)=\Delta\left(A(\varphi)-\varphi\left(x_{o}\right)\right)\left(x_{o}\right)
$$




$$
\begin{aligned}
& =\frac{\int_{M} \Delta_{x}(\chi(\rho(x, y)))\left(x_{0}\right)\left[\varphi(y)-\varphi\left(x_{0}\right)\right] d V(y)}{\int_{M} \chi\left(\rho\left(x_{0}, y\right)\right) d V(y)} \\
& -\frac{\int_{M} \chi\left(\rho\left(x_{o}, y\right)\right)\left[\varphi(y)-\varphi\left(x_{0}\right)\right] d V(y) \cdot \int_{M} \Delta_{x}\left(\chi(\rho(x, y))\left(x_{0}\right) d V(y)\right.}{\left\{\int_{M} \chi\left(\rho\left(x_{0}, y\right)\right) d V(y)\right\}^{2}} \\
& -2 \sum_{i=1}^{n} \frac{\int_{M}\left(e_{i}\right)_{x}(\chi(\rho(x, y)))\left(x_{0}\right) d V(y) \cdot \int_{M}\left(e_{i}\right)_{x}(\chi(\rho(x, y)))\left(x_{0}\right)\left[\varphi(y)-\varphi\left(x_{0}\right)\right] d V(y)}{\left\{\int_{M} \chi\left(\rho\left(x_{0}, y\right)\right) d V(y)\right\}^{2}} \\
& +2 \sum_{i=1}^{n} \frac{\left.\int_{M} \chi\left(\rho\left(x_{0}, y\right)\right)\left[\varphi(y)-\varphi\left(x_{0}\right)\right] d V(y) \cdot\left\{\int_{M}\left(e_{i}\right)\right)_{x}(\chi(\rho(x, y)))\left(x_{0}\right) d V(y)\right\}^{2}}{\left\{\int_{M} \chi\left(\rho\left(x_{0}, y\right)\right) d V(y)\right\}^{3}}
\end{aligned}
$$

where $\left\{e_{\imath} \mid i=1, \ldots, n\right\}$ is a local orthonormal frame around $x_{0}$ satisfying $\left(\nabla_{e l} e_{j}\right)\left(x_{o}\right)=0(i, j=1, \ldots, n)$ and the subscript $x$ means the differenciation with respect to $x$-variable. Therefore, we get

$$
|\Delta(A(\varphi))(x)| \leq 2 \cdot\left\{\frac{\int_{M} \mid \Delta_{x}(\chi(\rho(x, y)) \mid d V(y)}{\int_{M} \chi(\rho(x, y)) d V(y)}+2 n \frac{\left\{\int_{M}\left|\chi^{\prime}(\rho(x, y))\right| d V(y)\right\}^{2}}{\left\{\int_{M} \chi(\rho(x, y)) d V(y)\right\}^{2}}\right\}
$$

for all $x \in M$. Since $\Delta_{x}(\chi(\rho(x, y)))=\chi^{\prime}(\rho(x, y)) \cdot \Delta_{x} \rho(x, y)+\chi^{\prime \prime}(\rho(x, y))$, we obtain

(8) $|\Delta(A(\varphi))(x)| \leq 2\left\{f_{3}(x)+f_{2}(x)+2 n f_{1}(x)^{2}\right\} \times \sup _{y \in B_{x}(2)}|\varphi(y)-\varphi(x)|$, where

$$
\begin{aligned}
f_{1}(x) & =\frac{\int_{M}\left|\chi^{\prime}(\rho(x, y))\right| d V(y)}{\int_{M} \chi(\rho(x, y)) d V(y)} \\
f_{2}(x) & =\frac{\int_{M}\left|\chi^{\prime \prime}(\rho(x, y))\right| d V(y)}{\int_{M} \chi(\rho(x, y)) d V(y)}
\end{aligned}
$$

and 


$$
f_{3}(x)=\frac{\int_{M}\left|\chi^{\prime}(\rho(x, y))\right|\left|\Delta_{x} \rho(x, y)\right| d V(y)}{\int_{M} \chi(\rho(x, y)) d V(y)}
$$

Now, we shall estimate the part $\{\cdot\}$ in the inequality (8) from above in terms of a polynomial of $k(x)$. (We choose the sign of $k(x)$ to be nonnegative).

We shall begin with an estimate of $f_{1}$. When we represent the volume form $d V$ relative to geodesic porlar coordinates centered at $x$, we have $d V(y)=$ $a(t, \theta) d t d \theta$ for some positive function $a(t, \theta)$, where $d \theta$ is the standard volume form on the unit sphere $S_{x}^{n-1}(1) \subset T_{x} M$ and $t=\operatorname{dist}(x, y)$ and $\theta \in S_{x}^{n-1}(1)$. Thus, we get

$$
\int_{M}\left|\chi^{\prime}(\rho(x, y))\right| d V(y)=-\int_{M} \chi^{\prime}(\rho(x, y)) d V(y)=-\int_{S^{n-1}(1)} d \theta \int_{0}^{2} \chi^{\prime}(t) a(t, \theta) d t
$$

and for every fixed $\theta$

$$
\begin{gathered}
-\int_{0}^{2} \chi^{\prime}(t) a(t, \theta) d t=-[\chi(t) a(t, \theta)]_{t=\frac{1}{2}}^{t=2}+\int_{\frac{1}{2}}^{2} \chi(t) \cdot\left(\partial_{t} a\right)(t, \theta) d t \\
\leq a\left(\frac{1}{2}, \theta\right)+(n-1) k(x) \int_{\frac{1}{2}}^{2} \chi(t) \operatorname{coth}(k(x) t) a(t, \theta) d t \\
\leq a\left(\frac{1}{2}, \theta\right)+(n-1) k(x) \cdot \operatorname{coth}\left(\frac{k(x)}{2}\right) \cdot \int_{\frac{1}{2}}^{2} \chi(t) a(t, \theta) d t .
\end{gathered}
$$

In the above inequalities, we have used (1), (2) of $\chi$ and the Bishop-Gromov inequality, that is to say,

$$
0<\frac{n-1}{t} \leq \frac{\left(\partial_{t} a\right)(t, \theta)}{a(t, \theta)} \leq(n-1) k(x) \operatorname{coth}(k(x) t)
$$

for $t>0$ and $\theta \in S_{x}^{n-1}(1)$. Also we note that

$$
a\left(\frac{1}{2}, \theta\right) \leq 2 \int_{\frac{1}{2}}^{1} \chi(t) a(t, \theta) d t .
$$

Combining the inequalities above, we deduce

$$
\begin{aligned}
& f_{1}(x)=\frac{-\int_{S^{n-1}(1)} d \theta \int_{0}^{2} \chi^{\prime}(t) a(t, \theta) d t}{\int_{S^{n-1}(1)} d \theta \int_{0}^{2} \chi(t) a(t, \theta) d t} \\
& \leq 2+(n-1) k(x) \operatorname{coth}(k(x) / 2) \\
& \leq C_{1}^{\prime \prime}(k(x)+1),
\end{aligned}
$$


where $C_{1}{ }_{1}^{\prime \prime}$ is a positive constant depending only on $n=\operatorname{dim} M$.

Next, we shall estimate $f_{2}(x)$. We set

$$
C_{2}=\sup \left\{\left|\chi^{\prime}(t)\right|,\left|\chi^{\prime \prime}(t)\right| ; t \geq 0\right\} \text {. }
$$

Then, since $\chi^{\prime \prime} \geq 0$ on $[7 / 4,2]$, we see

$$
f_{2}(x) \leq \frac{C_{2} \cdot \operatorname{Vol}\left(B_{x}(7 / 4)\right)}{\int_{M} \chi(\rho(x, y)) d V(y)}+\frac{\int_{S^{n-1}(1)} d \theta \int_{\frac{7}{4}}^{2} \chi^{\prime \prime}(t) a(t, \theta) d t}{\int_{M} \chi(\rho(x, y)) d V(y)} .
$$

The first term on the right hand side in the above inequality is estimated as follows:

$$
\frac{C_{2} \cdot \operatorname{Vol}\left(B_{x}(7 / 4)\right)}{\int_{M} \chi(\rho(x, y)) d V(y)} \leq \frac{C_{2} \cdot \operatorname{Vol}\left(B_{x}(7 / 4)\right)}{\chi(7 / 4) \cdot \operatorname{Vol}\left(B_{x}(7 / 4)\right)}=\frac{C_{2}}{\chi(7 / 4)}=C_{3},
$$

where $C_{3}>0$ is a constant depending only on $\chi$.

With respect to the second term, we have

$$
\begin{gathered}
\int_{\frac{7}{4}}^{2} \chi^{\prime \prime}(t) a(t, \theta) d t=\left[\chi^{\prime}(t) a(t, \theta)\right]_{t=\frac{7}{4}}^{t=2}-\int \chi^{\prime}(t)\left(\partial_{t} a\right)(t, \theta) d t \\
\leq-\chi^{\prime}(7 / 4) a(7 / 4, \theta)-(n-1) k(x) \int_{\frac{7}{4}}^{2} \chi^{\prime}(t) a(t, \theta) \operatorname{coth}(k(x) t) d t \\
\leq-\chi^{\prime}(7 / 4) a(7 / 4, \theta)-(n-1) k(x) \operatorname{coth}(7 k(x) / 4) \cdot \int_{\frac{7}{4}}^{2} \chi^{\prime}(t) a(t, \theta) d t
\end{gathered}
$$

and, because $a(t, \theta)$ is an increasing function with respect $t>0$, we get

$$
\begin{aligned}
-\chi^{\prime}(7 / 4) a(7 / 4, \theta) & \leq C_{2} \cdot 8 \cdot \int_{\frac{7}{4}}^{\frac{15}{8}} a(t, \theta) d t \\
& \leq \frac{C_{2} \cdot 8}{\chi(15 / 8)} \cdot \int_{\frac{7}{4}}^{\frac{15}{8}} \chi(t) a(t, \theta) d t \\
& \leq C_{4} \int_{0}^{2} \chi(t) a(t, \theta) d t,
\end{aligned}
$$

where $C_{4}>0$ is a constant depending only on $\chi$. Hence, we obtain

$$
\begin{aligned}
f_{2}(x) \leq C_{3}+C_{4} & +(n-1) k(x) \operatorname{coth}(7 k(x) / 4) f_{1}(x) \\
\leq & C_{5}\left(k(x)^{2}+1\right)
\end{aligned}
$$


where $C_{5}>0$ is a constant depending only on $\chi$ and $n=\operatorname{dim} M$.

Now, it remains only to estimate the function $f_{3}$. The Bishop-Gromov inequality implies that for $y \in B_{x_{o}}(2)-B_{x_{o}}(1)$,

$$
\begin{aligned}
0 & <(n-1) \cdot \rho(x, y)^{-1} \\
& \leq\left(\Delta_{x} \rho(x, y)\right)(x) \\
& \leq(n-1) k(x) \operatorname{coth}(k(x) \rho(x, y)) \\
& \leq(n-1) k(x) \operatorname{coth} k(x) .
\end{aligned}
$$

Noting that $\left|\chi^{\prime}(\rho(x, y))\right|=0$ when $y \in B_{x}(1) \cup\left(M-B_{x}(2)\right)$, we see

$$
\begin{aligned}
& f_{3}(x) \leq(n-1) k(x) \operatorname{coth} k(x) \cdot f_{1}(x) \\
& \leq C_{6}\left(k(x)^{2}+1\right),
\end{aligned}
$$

where $C_{6}>0$ is a constant depending only on $n=\operatorname{dim} M$. Lemma 2.1 follows from the inequalities $(8) \sim(11)$.

Q. E. D.

\section{The proof of Theorem $\mathbf{1 . 2}$}

In the first place, we note that the following inequality holds by virtue of Lemma 2.1:

$$
\begin{aligned}
& \Delta\left\{A(\varphi)+C_{7}\left(\log \left(\rho+C_{8}\right)\right)^{-\varepsilon}\right\}(x) \\
& \leq-\frac{1}{\left(\rho+C_{8}\right)\left\{\log \left(\rho+C_{8}\right)\right\}^{\varepsilon+1}} \\
& \quad \times\left[C_{7} \varepsilon\left\{\Delta \rho-\frac{\varepsilon+1}{\left(\rho+C_{8}\right) \log \left(\rho+C_{8}\right)}-\left(\rho+C_{8}\right)^{-1}\right\}\right. \\
& \quad-C_{1}\left(k(x)^{2}+1\right)\left(\rho+C_{8}\right)\left\{\log \left(\rho+C_{8}\right)\right\}^{\varepsilon+1} \\
& \left.\quad \times \sup _{y \in B_{x}(2)}|\varphi(y)-\varphi(x)|\right]
\end{aligned}
$$

at $x$ for every positive constants $C_{7}, C_{8}$ and $\varepsilon$, where $\rho(\cdot)=\operatorname{dist}(o, \cdot)$ and $C_{1}$ is as in Lemma 2.1. Let us set

$$
K_{\max }(s)=\max \left\{K_{M}\left(\gamma_{v}^{\prime}(s) \wedge u\right) \mid u \in T_{\gamma_{v}(s)} M-\{0\}, \gamma_{v}^{\prime}(s) \perp u, v \in S_{o}^{n-1}(1)\right\},
$$

where $\gamma_{v}(s)=\exp _{o}(s v), S_{o}^{n-1}(1)=\left\{v \in T_{o} M \mid\|v\|=1\right\}$, and $\gamma_{v}^{\prime}(s) \wedge v$ is the 2-plane spanned by $\gamma_{v}^{\prime}(s)$ and $v$, and $K_{M}\left(\gamma_{v}(s) \wedge v\right)$ is its sectional curvature. Next we take a continuous function $K(s) \in C^{0}([0, \infty))$ such that

$$
K_{\max }(s) \leq K(s) \leq 0 \text { for all } s \in[0, \infty) .
$$


Using this function $K(s)$, we define $F_{K} \in C^{2}([0, \infty))$ to be a solution of the equation

$$
\left\{\begin{array}{c}
F_{K}^{\prime \prime}+K F_{K}=0 \\
F_{K}(0)=0, F_{K}^{\prime}(0)=1
\end{array}\right.
$$

Then, the comparison theorem implies

$$
\Delta \rho \geq(n-1) \frac{F_{K}^{\prime} \circ \rho}{F_{K} \circ \rho} \geq(n-1) \frac{1}{\rho}
$$

and

$$
S_{\rho} \geq \frac{F_{K}^{\prime} \circ \rho}{F_{K}^{\circ} \rho} \mathrm{Id}
$$

on $M-\{0\}$, where $S_{t}$ is the shape operator of $\partial B_{o}(t)=\{x \in M \mid \operatorname{dist}(o, x)=t\}$. Therefore, when $n=\operatorname{dim} M \geq 3$ and $C_{8}$ is sufficiently large, we have

$$
\Delta \rho-\frac{\varepsilon+1}{\left(\rho+C_{8}\right)\left\{\log \left(\rho+C_{8}\right)\right\}}-\frac{1}{\rho+C_{8}} \geq \frac{1}{2} \frac{F_{K}^{\prime} \circ \rho}{F_{K}^{\prime} \circ \rho} \quad \text { on } M-\{o\} .
$$

Also, it is easily seen that

$$
\sup _{y \in B_{x}^{(2)}}|\varphi(y)-\varphi(x)| \leq \frac{2 \cdot C_{9}}{F_{K}(\rho(x)-2)}
$$

for every $x \in M$ with $\rho(x) \geq 3$, where $C_{9}$ is a Lipschitz constant of $\varphi$ on $S_{o}^{n-1}(1)$ with standard sphere metric.

Hence, we obtain the following inequality:

$$
\begin{gathered}
\Delta\left\{A(\varphi)+C_{7}\left(\log \left(\rho+C_{8}\right)\right)^{-\varepsilon}\right\} \\
\leq-\frac{1}{\left(\rho+C_{8}\right)\left\{\log \left(\rho+C_{8}\right)\right\}^{\varepsilon+1}} \\
\times\left[\frac{C_{7} \cdot \varepsilon}{2} \frac{F_{K}^{\prime} \circ \rho}{F_{K} \circ \rho}-C_{9}^{\prime}\left(k^{2}+1\right) \frac{\left(\rho+C_{8}\right)\left\{\log \left(\rho+C_{8}\right)\right\}^{\varepsilon+1}}{F_{K}(\rho-2)}\right]
\end{gathered}
$$

on $M-B_{o}(3)$, where $C_{9}^{\prime}>0$ is a constant depending only on $\varphi$ and $C_{8}$ is arbitrary constant greater than or equal to $e^{2(\varepsilon+1)}$. The following is an immediate consequence of this inequality:

Lemma 3.1 (main lemma). Let $M$ be an Hadamard manifold of dimension $n \geq 3$. We fix a point $o$ in $M$ and define $k, K_{\max }, K$ and $F_{K}$ by (6), (13), (14) and (15), re- 
spectively. Suppose that there exist positive constants $C_{7}, \varepsilon$ and $t_{0} \geq 3$ such that

$$
\inf _{\operatorname{dist}(o, x) \geq t_{o}}\left\{C_{7} \frac{F_{K}^{\prime}(\rho(x))}{F_{K}(\rho(x))}-\frac{\left(k(x)^{2}+1\right)\left(\rho(x)+e^{2(\varepsilon+1)}\right)\left\{\log \left(\rho(x)+e^{2(\varepsilon+1)}\right)\right\}^{\varepsilon+1}}{F_{K}(\rho(x)-2)}\right\} \geq 0 .
$$

Then the asymptotic Dirichlet problem for the Laplace-Beltrami operator is solvable for any $\varphi \in C^{0}(S(\infty))$.

Proof. Without loss of generality, we may assume that $\varphi$ is a Lipschitz function on $S(\infty)=S_{o}^{n-1}(1)$ : (see [4]). Under the assumption of Lemma 3.1, we can find positive constants $C_{7}^{\prime}$ and $C_{8}$ so that $\varphi_{+}:=A(\varphi)+C_{7}^{\prime}\left(\log \left(\rho+C_{8}\right)\right)^{-\varepsilon}$ is superharmonic and $\varphi_{-}:=A(\varphi)-C_{7}^{\prime}\left(\log \left(\rho+C_{8}\right)\right)^{-\varepsilon}$ is subharmonic. We note that $\varphi_{+}$and $\varphi_{-}$are in $C^{2}(M-\{o\}) \cap C^{0}(\bar{M})$ and they have the same boundary data $\varphi$ on $S(\infty)$. The Perron method shows that there exists a harmonic function $u$ such that

$$
\varphi_{-} \leq u \leq \varphi_{+} \text {on } M .
$$

Therefore, $\Delta u=0, u \in C^{\infty}(M) \cap C^{0}(\bar{M})$ and $\left.u\right|_{s(\infty)}=\varphi$.

Q. E. D

We are now ready to prove Theorem 1.2 .

Let us assume that there exist constants $l>2$ and $\rho_{o}>0$ such that

$$
K_{M}\left(x_{0}\right) \leq-\frac{l(l-1)}{\rho^{2}} \text { for any } x_{o} \in M \text { satisfying } \operatorname{dist}\left(o, x_{o}\right)=\rho \geq \rho_{o} .
$$

Then, as a function $K(\rho)$ appeared in the inequality (14), we can take

$$
K(\rho)=-l(l-1) \rho^{-2} \text { for all } \rho \geq \rho_{o} .
$$

We respectively define functions $f$ and $B$ by

$$
f(\rho)=\left(\rho+C_{9}\right)^{l}
$$

and

$$
B(\rho)=\frac{f^{\prime}(\rho)}{f(\rho)}=\frac{l}{\rho+C_{9}} \text { for } \rho \geq \rho_{o},
$$

where $C_{9}>2$ is a positive constant determined so as to satisfy

$$
S_{\rho_{o}} \geq \frac{F_{K}^{\prime}\left(\rho_{o}\right)}{F_{K}\left(\rho_{o}\right)} \text { Id } \geq \frac{1}{\rho_{o}} \text { Id } \geq \frac{l}{\rho_{o}+C_{9}} \text { Id }=B\left(\rho_{o}\right) \text { Id }
$$

In the situation introduced above, we observe that 


$$
K(\rho) \leq-\frac{f^{\prime \prime}(\rho)}{f(\rho)} \text { for all } \rho \geq \rho_{o}
$$

and that $F_{K}$ and $f$ respectively satisfy the following Riccati equations:

$$
\left\{\frac{F_{K}^{\prime}(\rho)}{F_{K}(\rho)}\right\}^{\prime}+\left\{\frac{F_{K}^{\prime}(\rho)}{F_{K}(\rho)}\right\}^{2}+K(\rho)=0
$$

and

$$
B^{\prime}(\rho)+B^{2}(\rho)-\frac{f^{\prime \prime}(\rho)}{f(\rho)}=0 \text { for } \rho \geq \rho_{o}
$$

From (17), (18) and the Riccati equations, we obtain

$$
\frac{F_{K}^{\prime}(\rho)}{F_{K}(\rho)} \geq B(\rho)=\frac{l}{\rho+C_{9}} \text { for all } \rho \geq \rho_{o} .
$$

So,

$$
\left\{\frac{f}{F_{K}}\right\}^{\prime}(\rho)=\frac{f}{F_{K}}\left\{B-\frac{F_{K}^{\prime}}{F_{K}}\right\}(\rho) \leq 0
$$

Hence,

$$
\frac{1}{F_{K}}(\rho) \leq \frac{f\left(\rho_{o}\right)}{F_{K}\left(\rho_{o}\right)} \cdot \frac{1}{f(\rho)} \text { for } \rho \geq \rho_{o} \text {. }
$$

Now, we shall add the following assumption:

$$
\operatorname{Ric}_{M}(x) \geq-C_{10} \frac{\rho(x)^{l-2}}{(\log \rho(x))^{\theta+1}} \text { for } x \in M \text { satisfying } \rho(x) \geq \rho_{o},
$$

where $C_{10}$ and $\theta$ are positive constants. If two positive constants $C_{7}$ and $\rho_{0}$ are sufficiently large, and if $\varepsilon \in(0, \theta)$, then (19) and (21) imply that

$$
\begin{gathered}
C_{7} \frac{F_{K}^{\prime} \circ \rho}{F_{K}^{\circ} \rho}-\frac{k^{2}+1}{F_{K}(\rho-2)}\left(\rho+C_{8}\right)\left(\log \left(\rho+C_{8}\right)\right)^{\varepsilon+1} \\
\geq C_{7} \frac{l}{\rho+C_{9}}-\frac{f\left(\rho_{0}\right)}{F_{K}\left(\rho_{0}\right)} \frac{\rho+C_{8}}{\left(\rho+C_{9}-2\right)^{l}}\left\{\log \left(\rho+C_{8}\right)\right\}^{\varepsilon+1}\left\{C_{10} \frac{\rho^{l-2}}{(\log \rho)^{\theta+1}}+1\right\}>0
\end{gathered}
$$

on $M-B_{o}\left(\rho_{0}\right)$, where $C_{8}=e^{2(\varepsilon+1)}$. Therefore, under the assumption (16) and (21), the asymptotic Dirichlet problem for harmonic functions is solvable and we complete the proof of the case (a) in Theorem 1.2. 
Next, let us prove the case (b) in Theorem 1.2. We assume that $K_{M}(x) \leq$ $-a^{2}$ for all $x$ with $\operatorname{dist}(o, x) \geq \rho_{0}$. Let us take the constant $\rho_{1}>\rho_{0}$ and then define

$$
K(t)= \begin{cases}0, & t \in\left[0, \rho_{0}\right], \\ -\frac{a^{2}}{\rho_{1}-\rho_{0}}\left(t-\rho_{0}\right), & t \in\left[\rho_{0}, \rho_{1}\right], \\ -a^{2}, & t \in\left[\rho_{1}, \infty\right) .\end{cases}
$$

In addition we define $F_{K}$ to be the solution of equation (15). Then, we can prove that $F_{K}^{\prime}(t) / F_{K}(t) \rightarrow a$ as $t \rightarrow \infty$ and that $e^{a t} / F_{K}(t)$ converges to some positive constant as $t \rightarrow \infty$. Hence, applying Lemma 3.1 completes the proof of the case (b).

Remark 2. As is seen from the above proof, the assumption (b) in Theorem 1.2 can be weakened as follows: let us assume that ( $\left.b^{\prime}\right)$ there exist positive constants, $a, C$, and $\theta$ such that

$$
K_{M}(x) \leq-a^{2}
$$

and

$$
\operatorname{Ric}_{M}(x) \geq-C e^{a \rho(x)}(\rho(x)+1)^{-1}\{\log (\rho(x)+2)\}^{-1-\theta}
$$

for $x \in M$ outside a compact subset. Then we can solve the asymptotic Dirichlet problem.

Remark 3. For Hadamard manifolds with the sectional curvatures bounded between two negative constants, Anderson and Schoen [4] showed that the Martin boundary of the manifold conicides with the geometric boundary $S(\infty)$. But it is unclear that the Martin boundary of $M$ coincides with $S(\infty)$ under our assumption of Theorem 1.2 .

\section{A generalization}

When we consider the Dirichlet problem for harmonic maps, it is important to solve the problem for harmonic functions as a special case. Indeed, Avilés, Choi and Micallef's argument in [5] enables us to show the following:

THEOREM 4.1 (the Dirichlet problem for harmonic maps). Let $M$ be an Hadamard manifold satisfying the condition of Theorem 1.2 or more generally that of main lemma. $\overline{B_{\tau}(p)}$ denotes the closed geodesic ball of radius $\tau$ and center at $p$ in a complete 
$C^{\infty}$ Riemannian manifold $N$. We assume that $\tau<\min \{\pi / 2 \sqrt{\kappa}$, injectivity radius of $N$ at $p\}$, where $\kappa \geq 0$ is an upper bound for the sectional curvatures of $N$. Then for each $\phi \in C^{0}\left(S(\infty), \overline{B_{\tau}(p)}\right)$, there exists a unique $u \in C^{0}\left(\bar{M}, \overline{B_{\tau}(p)}\right) \cap C^{\infty}(M$, $\left.\overline{B_{\tau}(p)}\right)$ which is a harmonic map on $M$ and which equals $\phi$ on $S(\infty)$.

We note that when $N$ is an Hadamard manifold, there is no restriction on the upper bound of $\tau$ in Theorem 4.1 and therefore Theorem 1.2 is considered as a special case of Theorem 4.1. We remark that in [5], Avilés, Choi and Micallef solved the asymptotic Dirichlet problem for harmonic maps when the sectional curvatures of $M$ are bounded between two negative constants. But their arguments are also available in the case that $S(\infty)$ is regular for the Laplace-Beltrami operator, and we shall omit the proof of Theorem 4.1. (See also Akutagawa [1]).

\section{Examples}

The notion of the asymptotic Dirichlet problem on Hadamard manifolds is naturally extended to that on a manifold with a pole (see Choi [9]). (Let us recall that a point $p$ of a Riemannian manifold $N$ is called a pole if the exponential map $\exp _{p}: T_{p} N \rightarrow N$ is a diffeomorphism).

Definition 1 ([9]). Let $N$ be a Riemannian manifold with a fixed pole $p$. Given $v \in S_{p}^{n-1}(1)=\left\{w \in T_{p} N \mid\|w\|=1\right\}$, we define $\gamma_{v}$ to be the geodesic ray emanating the pole $p$ with $\gamma_{v}^{\prime}(0)=v$. Suppose $u$ is a function defined on $M$. We say that $u(q)$ converges to a number $A$ as $q \rightarrow \gamma_{v}(\infty)$ if for any $\varepsilon>0$ there exist $\delta>0$ and $r>0$ such that $|u(q)-A|<\varepsilon$ for all $q \in K(v, \delta, r)$. Here $K(v, \delta, r)$ is the truncated cone $\left\{\exp _{p}(t w) \in N \mid t>r, L_{p}(v, w)<\delta, w \in S_{p}^{n-1}(1)\right\}$.

Definition 2 (asymptotic Dirichlet problem with respect to a pole $p$ ([9])). Given $\phi \in C^{0}\left(S_{p}^{n-1}(1)\right)$, find a harmonic function on $N$ such that for every $v \in$ $S_{p}^{n-1}(1), u(q)$ converges to $\varphi(v)$ as $q \rightarrow \gamma_{v}(\infty)$ in the sense of Definition 1 .

Now, we shall make examples of a manifold with a pole which satisfies curvature conditions similar to those of Theorem 1.2, where the sectional curvatures shall be replaced by the radial curvatures with respect to the pole. We remark that the upper bound of the sectional curvatures in Theorem 1.2 can be replaced with the weaker hypothesis of the radial curvatures with respect to a pole of $M$, as is easily seen from the proof in Section 3.

Let $\xi$ be a unit Killing vector field on the standard unit sphere $\left(S^{2 n-1}, g_{0}\right)$ 
which satisfies $\operatorname{Ker} f_{*}=R \cdot \xi$ for the Hopf fibering $f: S^{2 n-1} \rightarrow C P^{n-1}$. We define a symmetric tensor $g_{h}$ on $S^{2 n-1}$ by $g_{h}=g_{0}-\omega_{\xi} \otimes \omega_{\xi}$, where $\omega_{\xi}$ is the 1 -form dual to $\xi$. We shall introduce the following Riemannian metric $G_{n \mu}$ on $R^{2 n}$ using two tensors $g_{h}$ and $\omega_{\eta} \otimes \omega_{\eta}$ :

$$
G_{\eta \mu}=d \rho^{2}+\eta(\rho)^{2} g_{h}+\mu(\rho)^{2} \omega_{\eta} \otimes \omega_{\eta},
$$

where $\rho$ is the Euclidean distance to the origin $O$ of $R^{2 n}$, and $\eta$ and $\mu$ are smooth function on $[0, \infty)$ satisfying

$$
\eta(0)=\mu(0)=0, \quad \eta^{\prime}(0)=\mu^{\prime}(0)=1,
$$

and

$$
\eta>0, \mu>0 \text { on }(0, \infty) .
$$

Some properties of the harmonic functions on $R_{\eta \mu}^{2 n}=\left(R^{2 n}, G_{\eta \mu}\right)$ are discussed in Kasue [17].

We shall assume that $n=2$ and choose $\eta$ and $\mu$ to make examples related to Theorem 1.2 .

Example 1. Let us take $\eta$ and $\mu$ such that

$$
\eta(\rho)=\rho^{l} \text { and } \mu(\rho)=\rho^{m} \text { for all } \rho \geq \rho_{0}>0 .
$$

We suppose $m>2 l$. Then, straightforward calculation shows

$$
\begin{gathered}
K_{R_{\eta \mu}^{4}}\left(X \wedge \partial_{\rho}\right) \leq-l(l-1) \rho^{-2}, \\
K_{R_{\eta \mu}^{4}}\left(X_{1} \wedge \partial_{\rho}\right)=-l(l-1) \rho^{-2}, \\
\operatorname{Ric}_{R^{4} \eta \mu}(q) \geq-C\left\{\rho^{2(m-2 l)}(q)+1\right\},
\end{gathered}
$$

and

$$
\frac{\operatorname{Ric}_{R_{\eta \mu}^{4}}\left(X_{1} \wedge X_{1}\right)}{\left\|X_{1}\right\|^{2}} \sim-2 \rho^{2(m-2 l)} \quad \text { as } \rho \rightarrow \infty
$$

for all $q \in R^{4}-\{O\}$, where $X$ is an arbitrary tangent vector at $q$ with $\operatorname{dist}(O, q)$ $=\rho$ and $X \perp \partial_{\rho}$, and where $X_{1}$ is any vector at $q$ satisfying $\operatorname{dist}(q, O)=\rho$, $X_{1} \perp \partial_{\rho}$ and $X_{1} \perp \tilde{\xi}$, and where $C>0$ is a constant which does not depend on $q$. Here, $\tilde{\xi}=(0, \xi)$ is the vector field on $(0, \infty) \times S^{3}=R^{4}-\{O\}$ that is canonically determined by the vector field $\xi$ on $S^{3}$. Therefore, if $8<4 l<2 m<5 l-2$, then this example satisfies curvature conditions similar to those of Theorem 1.2 (a), where $K_{M}$ should be replaced to be the radial curvatures with respect to the 
origin. As a matter of fact, if only $l, m>1$, the asymptotic Dirichlet problem with respect to the origin is solvable. (We note that this is not an Hadamard manifold).

Example 2. We set

$$
\begin{aligned}
& \eta(\rho)=a^{-1} \sinh (a \rho), \\
& \mu(\rho)=b^{-1} \sinh (b \rho),
\end{aligned}
$$

and assume $b>2 a>0$. Then,

$$
\begin{gathered}
K_{R_{\eta \mu}^{4}}\left(X \wedge \partial_{\rho}\right) \leq-a^{2}, \\
K_{R_{\eta \mu}^{4}}\left(X \wedge \partial_{\rho}\right)=-a^{2}, \\
\operatorname{Ric}_{R_{\eta \mu}^{4}}(q) \geq-C \cdot e^{2(b-2 a) \rho(q)},
\end{gathered}
$$

and

$$
\frac{\operatorname{Ric}_{R_{R_{\mu t}^{4}}^{4}}\left(X_{1}, X_{1}\right)}{\left\|X_{1}\right\|^{2}} \sim-8 \frac{a^{4}}{b^{2}} e^{2(b-2 a) \rho} \quad \text { as } \rho \rightarrow \infty
$$

for all $q \in R^{2}-\{O\}$, where $C>0$ is a constant depending only on $a$ and $b$. Hence, if $2 a<b<\frac{5}{2} a$, this example satisfies curvature conditions similar to those of Theorem 1.2 (b), where the condition $K_{R_{\eta \prime \prime}^{4}} \leq-a^{2}$ should be replaced to be radial curvatures with respect to the origin $\leq-a^{2}$. Actually, under the condition $a, b>0$, alone, the asymptotic Dirichlet problem with respect to the origin is solvable. (This example is also not an Hadamard manifold).

The following example may be somewhat interesting, because $\eta$ decreases exponentially but the asymptotic Dirichlet problem with respect to the origin is solvable.

Example 3. We take

$$
\eta(\rho)=\rho^{\frac{1}{2}}(\log \rho)^{\theta} e^{-\rho} \text { and } \mu(\rho)=\exp (\exp 2 \rho)
$$

for $\rho \geq \rho_{0}>0$ and suppose the constant $\theta$ satisfies $2 \theta>1$, then we can settle the asymptotic Dirichlet problem on $\left(R^{2 n}, G_{n \mu}\right)$ with respect to the origin. Indeed, given $\varphi \in C^{0}\left(S_{0}^{2 n-1}(1)\right)$, we extend it radially along the rays from the origin $O$ to a function on $R_{n \mu}^{2 n}-\{O\}$ and denote this function by $\tilde{\varphi}$. We may assume that $S_{0}^{2 n-1}(1)=\left\{v \in T_{0} R_{n \mu}^{2 n} \mid\|v\|=1\right\}$ has the standard sphere metric $g_{0}$ and that $\varphi$ is smooth with respect to the metric $g_{0}$. Then, we have 


$$
|\Delta \tilde{\varphi}|(x) \leq C\left\{\mu^{-2}(\rho(x))+\eta^{-2}(\rho(x))\right\}
$$

for all $x$ with $\operatorname{dist}(O, x)=\rho(x) \geq \rho_{0}$, where $C$ is a constant. Also, we get

$$
\Delta(\log \rho)^{-\varepsilon}=-\frac{\varepsilon}{\rho(\log \rho)^{\varepsilon+1}}\left\{(2 n-2) \frac{\eta^{\prime}(\rho)}{\eta(\rho)}+\frac{\mu^{\prime}(\rho)}{\mu(\rho)}-\frac{1}{\rho}-\frac{\varepsilon+1}{\rho \log \rho}\right\}
$$

for any $\varepsilon>0$. Consequently, if there exist positive constants, $\varepsilon, C^{\prime}$ and $\rho_{0}^{\prime}$ such that

$$
\max \left\{\eta^{-2}(\rho), \mu^{-2}(\rho)\right\} \leq \frac{C^{\prime}}{\rho(\log \rho)^{\varepsilon+1}}\left\{(2 n-2) \frac{\eta^{\prime}(\rho)}{\eta(\rho)}+\frac{\mu^{\prime}(\rho)}{\mu(\rho)}-\frac{1}{\rho}-\frac{\varepsilon+1}{\rho \log \rho}\right\}
$$

for all $\rho \geq \rho_{0}^{\prime}$, then we can solve the asymptotic Dirichlet problem on $\left(R^{2 n}, G_{\eta \mu}\right)$ with respect to the origin for any $\varphi \in C^{0}\left(S_{0}^{2 n-1}(1)\right)$. This condition (23) is actually satisfied in this case, Example 3 . Moreover, we should note the following two facts: first, when $2 \theta \leq 1$, there are no nonconstant bounded harmonic functions on $R_{\eta \mu}^{2 n}$; and secondly, for every $\theta \in R$, the conservative law for the heat equation does not hold on $R_{\eta \mu}^{2 n}$, where $\eta(\rho)=\rho^{\frac{1}{2}}(\log \rho)^{\theta} e^{-\rho}$ and $\eta(\rho)=$ $\exp (\exp 2 \rho)$ for $\rho \geq \rho_{0}>0$. (This example is also not an Hadamard manifold).

Remark 4. Kasue [17], gives the general condition which settle the asymptotic Dirichlet problem on $R_{\eta \mu}^{2 n}$ with respect to the origin. Indeed, he defined two integrals,

$$
I_{1}=\int_{1}^{\infty} \eta^{2 n-4}(r) \mu(r) \int_{r}^{\infty} \eta^{2-2 n}(s) \mu^{-1}(s) d s d r
$$

and

$$
I_{2}=\int_{1}^{\infty} \eta^{2 n-2}(r) \mu^{-1}(r) \int_{r}^{\infty} \eta^{2-2 n}(s) \mu^{-1}(s) d s d r
$$

and proved that

$\left(A_{1}\right)$ there are no nonconstant bounded harmonic functions on $R_{n \mu}^{2 n}$ if and only if $I_{1}=+\infty$;

$\left(A_{2}\right)$ the asymptotic Dirichlet problem with respect to the origin is solvable on $R_{\eta \mu}^{2 n}$ for any continuous function on $S_{O}^{2 n-1}(1)$ if and only if both $I_{1}$ and $I_{2}$ are finite;

$\left(A_{3}\right)$ when $I_{1}$ is finite but $I_{2}$ divergent, any bounded harmonic function is constant along each fiber of Hopf map $f$, and for a continuous function $\Psi$ on $C P^{n-1}$, there exists a unique bounded harmonic function $h$ on $R_{n \mu}^{2 n}$ such that $h(q)$ converges to $\Psi \circ f(v)$ as $q \rightarrow \gamma_{v}(\infty)$ for every $v \in S_{O}^{2 n-1}(1)$. 
Remark 5. While the author was preparing this paper, Professor Kasue informed the author that the following two condition $\left(B_{1}\right)$ and $\left(B_{3}\right)$ are equivalent;

$\left(B_{1}\right)$ Any bounded classical solution $u(x, t)$ of the Cauchy problem for the heat equation

$$
\begin{cases}\left(\partial_{t}-\Delta\right) u(x, t)=0 & \text { on } R_{n \mu}^{2 n} \times(0, T], \\ u(x, 0)=u_{0}(x) & \text { on } R_{\eta \mu}^{2 n},\end{cases}
$$

is uniquely determined by its initial data $u_{0}(x)$, where $T$ is a positive constant;

$\left(B_{2}\right) \int_{R_{\eta \mu}^{2 n}} p(x, y, t) d V_{\eta \mu}(y)=1$, where $p(x, y, t)$ is minimal heat kernel on $R_{\eta \mu}^{2 n}$ and $d V_{\eta \mu}$ is the Riemannian measure on $R_{\eta \mu}^{2 n}$;

$$
\left(B_{3}\right) \int_{0}^{\infty} I_{3}(t) d t=+\infty \text {, where } I_{3}(t)=\eta^{2-2 n}(t) \mu^{-1}(t) \int_{0}^{t} \eta^{2 n-2}(s) \mu(s) d s .
$$

The proof is not difficult. The fact that $\left(B_{1}\right)$ implies $\left(B_{2}\right)$ is obvious. To see $\left(B_{2}\right)$ implies $\left(B_{3}\right)$, it suffices to notice the following:

$$
\int_{\rho(x)}^{\infty} I_{3}(t) d t=\int_{R_{\eta \mu}^{2 n}} \int_{0}^{\infty} p(x, y, t) d t d V_{\eta \mu}(y)
$$

The equation (25) is easily proved from the fact that $\Theta(t) \equiv \int_{0}^{t} I_{3}(s) d s$ satisfies $\Delta(\Theta \circ \rho) \equiv 1$. Using the argument in Dodziuk [22, p.706 and p.707], we can show that $\left(B_{3}\right)$ implies $\left(B_{1}\right)$. In relation to the condition $\left(B_{1}\right)$, we remark that the classical solution $u$ of the Cauchy problem (24) satisfies

$$
\max _{R_{n \mu}^{2 n} \times[0, T]}|u(x, t)|=\max _{R_{n \mu}^{2 n}}|u(x, 0)|,
$$

if $\left(B_{3}\right)$ holds and if there exists monotone increasing sequence $\left\{R_{i}\right\}$ such that $N\left(R_{i}\right) / \Theta\left(R_{i}\right) \rightarrow 0$ as $R_{i} \rightarrow \infty$, where $N(s)=\sup \left\{|u(x, t)| ;(x, t) \in \partial B_{0}(s) \times\right.$ $[0, T]\}$ and where $\Theta(s)=\int_{0}^{s} I_{3}(t) d t$ and $\partial B_{O}(s)=\left\{x \in R_{\eta \mu}^{2 n} ; \rho(x)=s\right\}$ and $s$ is a positive constant.

\section{REFERENCES}

[1] K. Akutagawa, The Dirichlet problem at infinity for harmonic mapping between Hadamard manifolds, Geometry of Manifolds, ed. by K. Shiohama, Academic Press, 1989, 59-70.

[2] A. Ancona, Negatively curved manifolds, elliptic operators, and the Martin boundary, Ann. of Math., 125 (1987), 495-536. 
[3] M. T. Anderson, The Dirichlet problem at infinity for manifolds of negative curvature, J. Differential Geom., 18 (1983), 701-721.

[4] M, T. Anderson and R. Schoen, Positive harmonic functions on complete manifolds of negative curvature, Ann. of Math., 121 (1985), 429-461.

[5] P. Avilés, H. I. Choi and M. Micallef, Boundary behavior of Harmonic maps on negatively curved manifolds, J. Funct. Anal., 99 (1991), 293-331.

[6] R. Azencott, Behavior of diffusion semigroups at infinity, Bulletin Soc. Math. France, 102 (1974), 193-240.

[7] W. Ballmann, On the Dirichlet problem at infinity of manifolds of nonpositive curv. ature, Forum Math., 1 (1989), 201-213.

[8] S. Y. Cheng, The Dirichlet problem at infinity for nonpositively curved manifolds, Communications in Analysis and Geometry, 1 (1993), 101-112.

[9] H. I. Choi, Asymptotic Dirichlet problems for Harmonic functions on Riemannian manifolds, Trans. of Amer. Math. Soc., 281, 2, Feb., 1984.

[10] J. Dodziuk, Maximum principle for parabolic inequalities and the heat flow on open manifolds, Indiana Univ. Math. J., 32 (1983), 703-716.

[11] P. Eberlein and B. O'Neill, Visibility manifolds, Pacific J. Math., 46 (1973), 45-109.

[12] J. Eells and L. Lemaire, Selected topics in harmonic maps, C.B.M.S. Regional Conf. Series 50 (Amer. Math. Soc., Providence, R. I. 1983).

[13] J. Eelles and Lamaire, Another report on harmonic maps, Bull. London Math. Soc., 20 (1988), 385-524.

[14] P. Hsu, Brownian motion and Riemannian geometry, Contemp. Math. 73 (1988), 95-104.

[15] P. Hsu and P. March, The Limiting angle of certain Riemannian Brownian motions, Comm. on Pure Appl. Math., 38 (1985), 755-768.

[16] A. Kasue, Applications of Laplacian and Hessian comparison theorems, Advanced Studies in Pure Mathematics 3, Kinokuniya, Tokyo.

[17] A. Kasue, Harmonic functions of polynomial growth on complete manifolds, Proceeding of Symposia in Pure Mathematics, 54 (1993), Part 1.

[18] P. Li and S. T. Yau, On the parabolic kernel of the Schrōdinger operator, Acta Math., 156 (1986), 153-201.

[19] M. Murata, Uniqueness and non-uniqueness of the positive Cauchy problem for the heat equation on Riemannian manifolds, preprint.

[20] T. Sasaki, On the Green function of a complete Riemannian or Kāhlar manifold with asymptotically negative constant curvature and applications, Advanced Studies in Pure Mathematics 3, Kinokuniya, Tokyo.

[21] D. Sullivan, The Dirichlet problem at infinity for a negatively curved manifold, J. Differential Geom., 18 (1983), 723-732.

[22] S. T. Yau, Open problems in geometry, Proceeding of Symposia in Pure Mathematics, 54 (1993), Part 1, AMS.

\footnotetext{
Department of Mathematics

Faculty of Science

Osaka University

Toyonaka, Osaka 560

Japan
} 